\title{
Sustainable Development Goals- Post-2015 End Tuberculosis Strategy
}

\author{
*Mrs.Nandhini.M\&**Prof.Dr.KaralineKarunagari
}

\section{Abstract:}

Tuberculosis (TB) is caused by bacteria (Mycobacterium tuberculosis) that most often affect the lungs. People infected with TB bacteria have a 10\% lifetime risk of falling ill with TB. Tuberculosis (TB) is a top infectious disease killer worldwide. Over 95\% of TB deaths occur in low-and middle-income countries, and it is among the top 5 causes of death for women aged 15 to 44.1Transforming our world: the 2030 agenda for sustainable development. It outlines global impact targets to reduce TB deaths by 90\% and to cut new cases by 80\% between 2015 and 2030, and to ensure that no family is burdened with catastrophic costs due to TB. WHO has gone one step further and set a 2035 target of 95\% reduction in deaths and a 90\% decline in TB incidence similar to current levels in low TB incidence countries today2.SDGs and End TB Strategy implementation will require very active engagement of all sectors and partners, from the highest level government officials to civil society. The ultimate goal of TB elimination requires that appropriate interventions are implemented everywhere and the underlying drivers are not only addressed, but sustainably addressed ${ }^{3}$.

\section{Keywords: Tuberculosis,Sustainable Development Goals and Tuberculosis strategy}

\section{INTRODUCTION}

Tuberculosis (TB) is caused by bacteria (Mycobacterium tuberculosis) that most often affect the lungs. Tuberculosis is curable and preventable. TB is spread from person to person through the air. People infected with TB bacteria have a 10\% lifetime risk of falling ill with TB. However, persons with compromised immune systems, such as people living with HIV, malnutrition or diabetes, or people who use tobacco, have a much higher risk of falling ill. People with active TB can infect 10-15 other people through close contact over the course of a year. Without proper treatment, $45 \%$ of HIV-negative people with TB on average and nearly all HIV-positive people with TB will die. ${ }^{1}$

\section{MAGNITUDE OF THE PROBLEM:}

Tuberculosis (TB) is a top infectious disease killer worldwide. In 2014, 9.6 million people fell ill with TB and 1.5 million died from the disease. Over $95 \%$ of TB deaths occur in lowand middle-income countries, and it is among the top 5 causes of death for women aged 15 to 44.In 2014, an estimated 1 million children became ill with TB and 140000 children died 
of TB. TB is a leading killer of HIV-positive people: in 2015, 1 in 3HIV deaths was due to TB. Globally in 2014, an estimated 480 000 people developed multidrug-resistant TB (MDR-TB). The Millennium Development Goal target of halting and reversing the TB epidemic by 2015 has been met globally. TB incidence has fallen by an average of $1.5 \%$ per year since 2000 and is now $18 \%$ lower than the level of 2000.The TB death rate dropped $47 \%$ between 1990 and 2015.An estimated 43 million lives were saved through TB diagnosis and treatment between 2000 and 2014. Ending the TB epidemic by 2030 is among the health targets of the newly adopted Sustainable Development Goals. ${ }^{1}$

\section{"TRANSFORMING OUR WORLD: THE 2030 AGENDA FOR SUSTAINABLE DEVELOPMENT"}

In 2015, September 25 countries were adopted and set of goals to end poverty, protect the planet, and ensure prosperity for all as part of a new sustainable development agenda. Each goal has specific targets to be achieved over the next 15 years. For the goals to be reached, everyone needs to do their part: Governments, the private sector, civil society and energetic people.

In 2015 set as the target year for the Millennium Development Goals (MDGs),a new agenda is needed to address the three interconnected elements of sustainable development: economic growth, social inclusion and environmental sustainability ${ }^{1}$

\section{POST-2015 END TUBERCULOSIS STRATEGY}

In 2014 May 19, the 67th World Health Assembly (WHA) adopted WHO's "Global strategy and targets for tuberculosis prevention, care and control after 2015". This post-2015 global tuberculosis strategy, labeled the End TB Strategy, was shaped during the past 2 years. A wide range of stakeholders from ministries of health and national tuberculosis programmes to technical and scientific institutions, financial and development partners, civil society and health activists, non-governmental organizations, and the private sector contributed to its development. The strategy has vision on making the world free of tuberculosis, with zero deaths, disease and suffering due to the disease. ${ }^{1 \& 2}$

\section{WHO RESPONSE:}

WHO pursues 6 core functions in addressing TB.

- Provide global leadership on matters critical to TB.

- Develop evidence-based policies, strategies and standards for TB prevention, care and control, and monitor their implementation.

- Provide technical support to Member States, catalyze change, and build sustainable capacity.

- Monitor the global TB situation, and measure progress in TB care, control, and financing.

- Shape the TB research agenda and stimulate the production, translation and dissemination of valuable knowledge.

- Facilitate and engage in partnerships for TB action.

The WHO End TB Strategy, adopted by the World Health Assembly in May 2014, is a blueprint for countries to end the TB epidemic by driving down TB deaths, incidence and eliminating catastrophic costs. It outlines global impact targets to reduce TB deaths by $90 \%$ and to cut new cases by $80 \%$ between 2015 and 2030, and to ensure that no family is burdened with catastrophic costs due to TB. Ending the TB epidemic by 2030 is among the health targets of the newly adopted Sustainable Development Goals. WHO has gone one step further and set a 2035 target of $95 \%$ reduction in deaths and a $90 \%$ decline in TB incidence - similar to current levels in low TB incidence countries today. 
The Strategy outlines 3 strategic pillars that need to be put in place to effectively end the epidemic:

- Pillar 1: integrated patient-centered care and prevention

- Pillar 2: bold policies and supportive systems

- Pillar 3: intensified research and innovation.

The success of the Strategy will depend on countries respecting the following 4 key principles as they implement the interventions outlined in each pillar:

- Government stewardship and accountability, with monitoring and evaluation

- Strong coalition with civil society organizations and communities

- Protection and promotion of human rights, ethics and equity

- Adaptation of the strategy and targets at country level, with global collaboration

WHO End TB Strategy and Targets with SDG:

Global development has entered a new era. World leaders are committing to 'end poverty, promote prosperity and people's well-being while protecting the environment by 2030'.Seventeen new Sustainable Development Goals (SDGs) were adopted at the United Nations Summit in September 2015. The SDG era began on 1 January 2016; at the same time that the WHO's new End TB Strategy to prevent, control and end the TB epidemic came into action. The SDGs build on the unfinished Millennium Development Goals (MDGs) agenda, but with a broader scope and more ambitious targets. They cover three dimensions: economic, social and environmental. The SDGs are 'Universal, indivisible and interlinked', and the interdependence between health and development as an integral part .
The overriding vision of the SDGs is that 'all human beings can fulfill their potential in dignity and equality and in a healthy environment' and 'enjoy prosperous and fulfilling lives.

There are multiple links between TB and poverty and food security (Goals $1 \& 2$ ). Preventing lost work hours due to TB globally to achieving sustainable economic growth, and productive employment (Goal 8). When the world strengthens enforceable legislation for the promotion of gender equality (Goal 5) and reduces inequalities by eliminating discriminatory practices (Goal 10), people will be able to access TB diagnosis and care more easily in cases where financial inequity, family responsibilities and cultural barriers may have prevented them from receiving care in the past.

The specific SDG 'Health Goal' (Goal No. 3) aims to 'ensure healthy lives and promote well-being for all at all ages. Target No. 3.3 as 'End the epidemics of AIDS, tuberculosis, malaria and neglected tropical diseases and combat hepatitis, water-borne diseases and other communicable diseases'. he End TB Strategy has a longer timeline than the SDGs, up to 2035. Although the 80\%/90\% reduction represents 2030 milestones in the End TB Strategy, the targets are $90 \%$ incidence and $95 \%$ death rate reduction by 2035 , which means that the global burden of TB should be similar to the burden in low-incidence countries today. This is a substantial shift in ambition level compared to the MDG era (and the related global TB strategy), which aimed solely at reverting the previous upward incidence trend within a 15-year horizon. In short, the End TB Strategy targets cannot be achieved unless rapid and substantial progress is made towards the SDGs. Conversely, better TB care and prevention will help achieve the SDGs in several ways. ${ }^{3}$

A crucial health target is No 3.8: 'Achieve universal health coverage, including financial risk protection, access to quality essential health care services and access to safe, effective, quality and affordable essential 
medicines and vaccines for all'. TB coverage indicators (TB detection and treatment success) are part of the metrics to monitor progress towards this target, given they are recognized as an essential health intervention for a disease that disproportionately affects the poorest. The End TB Strategy indicator on 'catastrophic costs due to TB' could complement monitoring of the progress towards social protection too. In the background analysis for the SDGs, TB diagnosis and treatment were listed among the most cost-effective health interventions, and also one that directly contributes to improved productivity and, therefore, overall societal development ${ }^{3}$.

TB as contributor to development is thus well captured in the SDGs. It is not difficult to justify the fact that TB can also benefit from the SDG achievements. Examples of actions that would address key TB determinants are clear-cut in the new goals: ending poverty in all its forms everywhere; ending hunger, achieving food security and improved nutrition, and promoting sustainable agriculture; ensuring inclusive and equitable quality education and promoting lifelong learning opportunities for all; achieving gender equality; ensuring access to affordable, reliable, sustainable and modern energy for all; reducing inequality within and among countries; and making cities and human settlements inclusive, safe, resilient and sustainable. ${ }^{3}$

One theme that could have been better emphasized in the SDGs is health research. Goal 9 includes to 'foster innovation' and one of the Goal 3 targets is about 'strengthening research for medicines and vaccines'. However, investments in broader health research, including epidemiology, social science and health systems research, and the required infrastructure, training and career paths are not clearly outlined.

Without it, the path forward will be winding. The third pillar of the End TB strategy addresses this need, and a global action framework has been developed to foster its implementation, promote capacity building in TB endemic countries and stimulate multidisciplinary research along the continuum from basic science to operational research. ${ }^{3}$

The SDGs promise to strengthen the required institutional, infrastructure and partnership foundations to make goals achievable. Goal $\mathbf{1 6}$ is to 'Promote peaceful and inclusive societies for sustainable development, provide access to justice for all and build effective, accountable and inclusive institutions at all levels', and Goal 17 is to 'Strengthen the means of implementation and revitalize the global partnership for sustainable development'. The keywords are there: freedom, peace, security, human rights, rule of law, good governance, and commitment to just and democratic societies. It is up to all of us to contribute to the realization of this vision and sustain it. ${ }^{3}$

\section{CONCLUSION:}

SDGs and End TB Strategy implementation will require very active engagement of all sectors and partners, from the highest level government officials to civil society. This is why WHO makes a point of defining the target audience for the End TB Strategy in much broader terms than for previous global TB strategies. The ultimate goal of TB elimination (less than one TB case per million population) requires that appropriate interventions are implemented everywhere and the underlying drivers are not only addressed, but sustainably addressed. History teaches us that epidemics re-emerge when neglect, ignorance and carelessness are let to prevaricate commitment, science and care. Being ahealth personnel it is our responsibility to take it as a challenge to reach end to the disease Tuberculosis. ${ }^{1,2 \& 3}$

\section{REFERENCES:}

1.WHO. Documentation for World Health Assembly 67.http://apps.who.int/gb/ebwha/pdf_files/WHA6 7/A67_11-en.pdf (accessed Sep 18, 2016).

2.WHO. Global tuberculosis control: WHO report 2016 (WHO/HTM/TB/accessed sep 18, 2016.). Geneva: World Health Organization, 2016.

3. Global TB Programme, World Health Organization, Geneva, Switzerland. http://dx.doi.org/10.1016/S0140-6736(15)605700 (accessed Sep 18, 2016) 